\title{
Commentary: Still a leaking problem: Questions remain in the management of ischemic mitral regurgitation
}

\author{
Maria Servito, BHSc, ${ }^{\mathrm{a}}$ Malak Elbatarny, MD, ${ }^{\mathrm{b}}$ and \\ Stephen E. Fremes, MD, MSc, FRCSC, FACP, FACC ${ }^{c}$
}

Ischemic mitral regurgitation (IMR) is associated with a substantial increase in late mortality, even when the severity is mild. ${ }^{1}$ The wide variability of clinical presentation, functional characteristics, and surgical and interventional treatment modalities renders clinical decision making challenging and controversial. Nappi and colleagues ${ }^{2}$ address this dilemma in a study-level meta-analysis comparing long-term outcomes of various treatment approaches to IMR.

Data from 12 studies comparing various management techniques for IMR were aggregated ( 8 randomized trials and 4 propensity-matched studies; $\mathrm{N}=2848$ ). Relevant, clinically important outcomes were reported: early and long-term all-cause mortality, rehospitalization, reoperation, and composite adverse events. The study performed pairwise comparisons of guideline-directed medical therapy (GDMT), MitraClip (Abbott Laboratories, Abbott Park, Ill), coronary artery bypass grafting (CABG), surgical mitral valve replacement (MVR) and surgical mitral valve repair (MVr), mostly restrictive mitral annuloplasty (RMA) but also RMA with subvalvular interventions (SVI). Figure 1 summarizes the key findings of the study. A potential caveat of the review is the risk of a type 1 error

From the ${ }^{\mathrm{a} S} \mathrm{School}$ of Medicine, Queen's University, Kingston, Ontario, Canada; ${ }^{\mathrm{b}} \mathrm{Di}$ vision of Cardiac Surgery, Department of Surgery, University of Toronto, Toronto, Ontario, Canada; and ${ }^{\mathrm{c} S}$ chulich Heart Centre and Division of Cardiac Surgery, Department of Surgery, Sunnybrook Health Sciences Centre, University of Toronto, Toronto, Ontario, Canada.

Disclosures: The authors reported no conflicts of interest.

The Journal policy requires editors and reviewers to disclose conflicts of interest and to decline handling or reviewing manuscripts for which they may have a conflict of interest. The editors and reviewers of this article have no conflicts of interest.

Received for publication June 28, 2020; revisions received June 28, 2020; accepted for publication June 28, 2020; available ahead of print July 13, 2020.

Address for reprints: Stephen E. Fremes, MD, MSc, FRCSC, FACP, FACC, Schulich Heart Centre and Division of Cardiac Surgery, Department of Surgery, Sunnybrook Health Sciences Centre, 2075 Bayview Ave, Room H4 05, Toronto, Ontario, Canada M4N 3M5 (E-mail: stephen.fremes@ sunnybrook.ca).

J Thorac Cardiovasc Surg 2022;163:626-8

$0022-5223 / \$ 36.00$

Copyright (c) 2020 by The American Association for Thoracic Surgery

https://doi.org/10.1016/j.jtcvs.2020.06.113

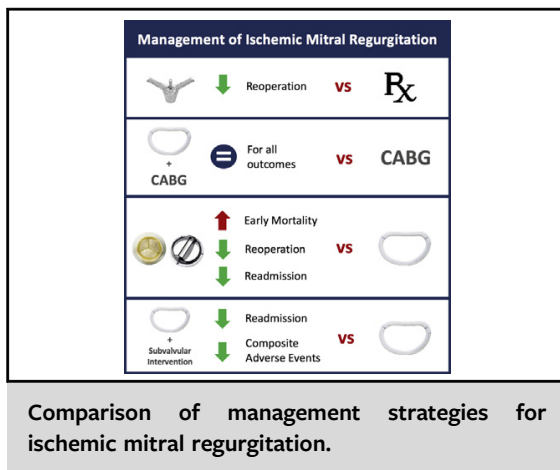

CENTRAL MESSAGE

Questions remain regarding

optimal management strategies

for ischemic mitral regurgitation

based on clinical and anatomical

features.

from multiple hypothesis testing. There were 4 pairwise treatment comparisons with 5 separate end points for each comparison; that is, 20 individual statistical comparisons.

As the authors note, limited robust evidence renders this analysis still insufficient to derive specific management recommendations. In fact, most comparisons consisted of 3 or fewer studies; only the CABG + RMA versus CABG comparison was based on 5 studies. Although a quantitative synthesis of the data is essential to understand treatment effects with large numbers of studies, one can argue that a narrative review may be more informative with limited data. The MitraClip + GDMT versus GDMT comparison consisted of only 2 studies: 1 drove the results with double the number of patients and each studied either proportionate or disproportionate mitral regurgitation, which raises concerns about the appropriateness of pooled comparison due to clinical heterogeneity. ${ }^{3,4}$ Before randomization, Cardiovascular Outcomes Assessment of the MitraClip Percutaneous Therapy patients received medical therapy titrated to maximally tolerated doses, potentially inducing reverse left ventricular (LV) remodeling and thus, smaller LV volumes disproportionate to mitral regurgitation severity. In contrast, Multicentre Study of Percutaneous Mitral Valve Repair MitraClip Device in Patients With Severe Secondary Mitral Regurgitation patients unlikely received similarly aggressive forms of medical management, thus resulting in LV volumes proportionate to mitral regurgitation severity. ${ }^{3}$ Although the meta-analysis results suggest that MitraClip does not provide important clinical advantages, the 


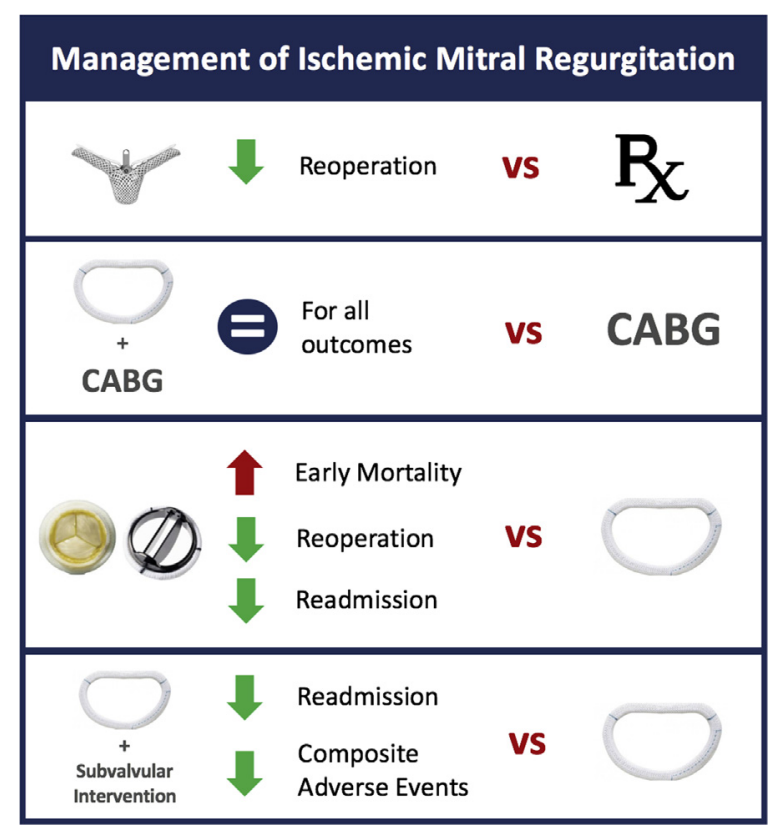

FIGURE 1. Management for ischemic mitral regurgitation. $R x$, Prescription; $C A B G$, coronary artery bypass grafting.

conclusions of the 2 individual studies were markedly different, ${ }^{3,4}$ consistent with the statistical heterogeneity for all outcomes except reoperation. Similarly, severe mitral regurgitation was not excluded from the $\mathrm{CABG}+\mathrm{RMA}$ versus $\mathrm{CABG}$ comparison despite the lack of equipoise about isolated revascularization in severe mitral regurgitation. ${ }^{5}$ Additionally, the lack of differentiation between acute and chronic presenting patients makes interpretation challenging. Although viability investigations can differentiate between cases of moderate mitral regurgitation requiring CABG + RMA or CABG alone, ${ }^{6}$ these data were not available for comparison.

The authors report reduced reoperation following MitraClip + GDMT versus GDMT (odds ratio [OR], $0.40 ; 95 \%$ confidence interval $[\mathrm{CI}], 0.22-0.72 ; P=.003$ ), and there was limited heterogeneity with this comparison $\left(I^{2}=22 \%\right) .^{2}$ Experience with reoperation after MitraClip is in its infancy ${ }^{7}$ because these patients represent poor surgical candidates at the time of presentation, much less in the event of late failure. Ultimately, neither reduced reoperation nor the symptomatic benefit reported in individual studies translated to late survival benefit. ${ }^{2}$ Evidence of mortality benefit of MitraClip is currently limited to pooled observational comparisons in the literature. ${ }^{8}$

Although included studies comparing CABG + RMA versus $\mathrm{CABG}$ individually reported improvements in New York Heart Association functional class, mitral regurgitation grade, and LV dimensions, ${ }^{9,10}$ these did not translate to any difference in mortality, rehospitalization, or composite adverse events in the meta-analysis. ${ }^{2}$ This absence of benefit in hard clinical end points despite improvements in echocardiographic and symptomatic parameters is not intuitive and requires further study. The comparison of $\mathrm{CABG}+\mathrm{RMA}$ versus $\mathrm{CABG}$-despite being based on 5 individual studies-also still has not settled the controversy of optimal surgical strategy for moderate IMR. The comparison of RMA + SVI versus RMA consisted of only 2 studies; but still, pooled readmission and the composite end point were significantly in favor of SVI. The highly significant difference in the composite end point (OR, 0.3; 95\% CI, 0.12-0.74; $P=.009$; $I^{2}=28 \%$ ) suggests this comparison may be underpowered to detect potential promise of SVI techniques in other clinically relevant end points.

We commend the authors for performing a meta-analysis of MVR versus MVr, a topic lacking strong recommendations due to contradicting evidence. An increase in hospital mortality was observed in MVR versus $\mathrm{MVr}$ (OR, 1.91; $95 \%$ CI, 1.18-3.12; $P=.009 ; I^{2}=0 \%$ ). However, this must be interpreted with caution because unadjusted results of a study with significantly more urgent cases in MVR than $\mathrm{MVr}$ were analyzed, possibly influencing the early outcomes to favor the latter. The validity of pooling the results must also be questioned due to the heterogeneity in baseline LV end-diastolic dimension between studies. The reported baseline LV end-diastolic dimension for all studies was $<6.5 \mathrm{~cm}$, which is the recommended threshold for MVR in the guidelines. ${ }^{11}$ Still, late outcomes of MVR, such as late reoperation (OR, 0.60; 95\% CI, 0.36-1.00; $P=.05$; $\left.I^{2}=0 \%\right)$ and rehospitalization $(\mathrm{OR}, 0.45 ; 95 \% \mathrm{CI}, 0.23-$ $0.87 ; P=.02 ; I^{2}=69 \%$ ), were superior. MVr will likely never match prosthetic valve durability and lacks universal applicability. Nevertheless, careful selection of $\mathrm{MVr}$ technique may provide the key to optimal early and late outcomes. For example, patients with advanced LV remodeling and MV deformation are typically nonresponders to isolated RMA, potentially requiring concomitant SVI. ${ }^{12}$ Yet, all included studies comparing MVr with MVR primarily employed RMA as a repair technique. In general, prospective trials on SVI are limited, with evidence mostly being derived from observational studies and small case series. Consequently, no guidelines or standardization exist, generalizability of good outcomes remains unclear, and anecdotally, uptake is limited. A comparison of MVR versus RMA + SVI in severe IMR may actually provide more valuable insights rather than MVR versus isolated RMA.

Many different treatment approaches for IMR exist. To elucidate choice of optimal strategy, a multidisciplinary heart team must be involved, particularly considering the importance of GDMT as an adjunct or primary strategy. We congratulate Nappi and colleagues ${ }^{2}$ for this elegant compilation of the existing evidence on this complex topic. This work emphasizes the need for granular randomized data stratified by clinical and pathological variants of IMR. 


\section{References}

1. Lamas GA, Mitchell GF, Flaker GC, Smith SC Jr, Gersh BJ, Basta L, et al. Clinical significance of mitral regurgitation after acute myocardial infarction. Circulation. 1997;96:827-33

2. Nappi F, Antoniou GA, Nenna A, Michler R, Benedetto U, Avtaar Singh SS, et al Treatment options for ischemic mitral regurgitation: a meta-analysis. $J$ Thorac Cardiovasc Surg. 2022;163:607-22.e14.

3. Stone GW, Lindenfeld JA, Abraham WT, Kar S, Lim DS, Mishell JM, et al. Transcatheter mitral-valve repair in patients with heart failure. $N$ Engl J Med. 2018;379:2307-18.

4. Obadia JF, Messika-Zeitoun D, Leurent G, Iung B, Bonnet G, Piriou N, et al. Percutaneous repair or medical treatment for secondary mitral regurgitation. $N$ Engl J Med. 2018;379:2297-306.

5. Mihaljevic T, Lam BK, Rajeswaran J, Takagaki M, Lauer MS, Gillinov AM, et al. Impact of mitral valve annuloplasty combined with revascularization in patients with functional ischemic mitral regurgitation. J Am Coll Cardiol. 2007;49:2191-201.

6. Penicka M, Linkova H, Lang O, Fojt R, Kocka V, Vanderheyden M, et al. Predictors of improvement of unrepaired moderate ischemic mitral regurgitation in patients undergoing elective isolated coronary artery bypass graft surgery. Circulation. 2009;120:1474-81.

7. Argenziano M, Skipper E, Heimansohn D, Letsou GV, Woo YJ, Kron I, et al. Surgical revision after percutaneous mitral repair with the MitraClip device. Ann Thorac Surg. 2010;89:72-80.
8. Bertaina M, Galluzzo A, D'Ascenzo F, Conrotto F, Grosso Marra W, Frea S, et al. Prognostic impact of MitraClip in patients with left ventricular dysfunction and functional mitral valve regurgitation: a comprehensive meta-analysis of RCTs and adjusted observational studies. Int J Cardiol. 2019;290:70-6

9. Fattouch K, Guccione F, Sampognaro R, Panzarella G, Corrado E, Navarra E, et al. POINT: efficacy of adding mitral valve restrictive annuloplasty to coronary artery bypass grafting in patients with moderate ischemic mitral valve regurgitation: a randomized trial. J Thorac Cardiovasc Surg. 2009;138: $278-85$.

10. Chan KMJ, Punjabi PP, Flather M, Wage R, Symmonds K, Roussin I, et al. Coronary artery bypass surgery with or without mitral valve annuloplasty in moderate functional ischemic mitral regurgitation: final results of the Randomized Ischemic Mitral Evaluation (RIME) trial. Circulation. 2012;126: 2502-10.

11. AATS Ischemic Mitral Regurgitation Consensus Guidelines Writing Committee, Kron IL, LaPar DJ, Acker MA, Adams DH, Ailawadi G, Bolling SF, et al. 2016 update to the American Association for Thoracic Surgery (AATS) consensus guidelines: ischemic mitral valve regurgitation. J Thorac Cardiovasc Surg. 2017; 153:e97-114.

12. Mihos CG, Santana O. Mitral valve repair for ischemic mitral regurgitation: lessons from the Cardiothoracic Surgical Trials Network randomized study. J Thorac Dis. 2016;8:E94-9. 